\title{
Expanded Endoscopic Endonasal Resection of Retrochiasmatic Craniopharyngioma
}

\author{
Justin R. Davanzo ${ }^{1}$ Neerav Goyal ${ }^{2} \quad$ Brad E. Zacharia ${ }^{1}$ \\ ${ }^{1}$ Department of Neurological Surgery, Penn State Health Milton S. \\ Hershey Medical Center, Hershey, Pennsylvania, United States \\ ${ }^{2}$ Department of Surgery, Division of Otolaryngology, Penn State \\ Health Milton S. Hershey Medical Center, Hershey, Pennsylvania, \\ Address for correspondence Justin R. Davanzo, MD, Department of \\ Neurological Surgery, Penn State Health Milton S. Hershey Medical \\ Center, 30 Hope Dr., EC 110, Hershey, PA 17033, United States \\ (e-mail: jdavanzo@pennstatehealth.psu.edu).
} United States

J Neurol Surg B 2018;79(suppl S2):S194-S195.

\begin{abstract}
Keywords

- craniopharyngioma

- neuro-oncology

- skull base

- endoscopic surgery

This video abstract demonstrates the use of the expanded endoscopic endonasal approach for the resection of a retrochiasmatic craniopharyngioma. These tumors are notoriously difficult to treat, and many approaches have been tried to facilitate safe and effective resection. The endoscopic endonasal approach has been increasingly utilized for selected sellar/suprasellar pathology. We present the case of a 39-year-old man who was found to have a cystic, partially calcified suprasellar mass consistent with a craniopharyngioma. To facilitate robust skull base repair, a vascularized nasoseptal flap was harvested. A wide sphenoidotomy was performed and the sella and tuberculum were exposed. After the dural opening and arachnoid dissection, the stalk was identified, merging seamlessly with the tumor capsule. The lesion was then internally debulked with the use of an ultrasonic aspirator. The capsule was then dissected off of the optic chiasm, thalamus, and hypothalamus. The cavity was inspected with an angled endoscope to ensure complete resection. A multilayered reconstruction was performed using autologous fascia lata, the previously harvested nasoseptal flap, and dural sealant. Postoperatively, the patient did have expected panhypopituitarism but remained neurologically intact and had improvement in his vision. In conclusion, this video demonstrates how an expanded endonasal approach can be used to safely resect a craniopharyngioma, even when in close proximity to delicate structures such as the optic chiasm.

The link to the video can be found at: https://youtu.be/tahjHmrXhc4.
\end{abstract}

Conflict of Interest

None.

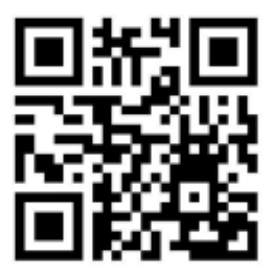

www.thieme.com/skullbasevideos

www.thieme.com/jnlsbvideos received

October 15, 2017

accepted

December 7, 2017

published online

January 23, 2018
DOI https://doi.org/

10.1055/s-0038-1623524.

ISSN 2193-6331. (c) 2018 Georg Thieme Verlag KG

Stuttgart · New York
License terms

(c) (i) $\ominus$ (\$) 


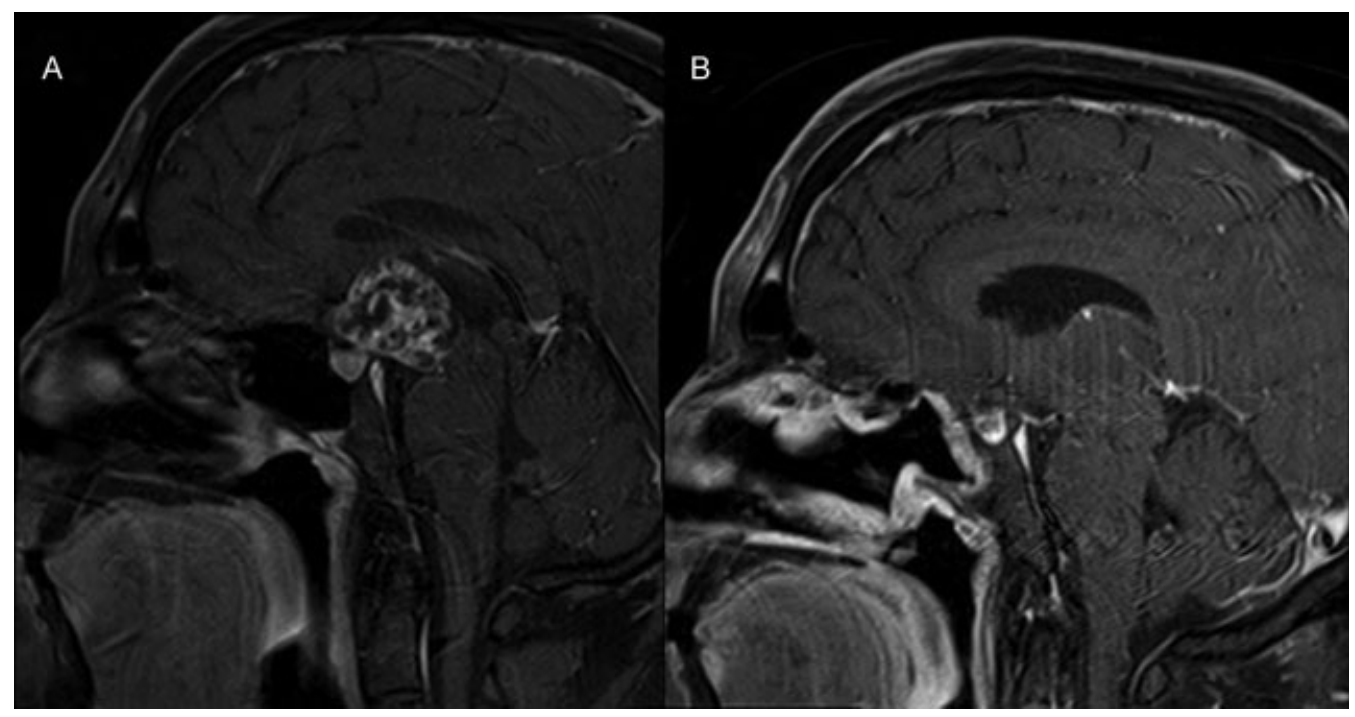

Fig. 1 Preoperative (A) and postoperative (B) sagittal magnetic resonance images showing suprasellar lesion and gross total resection.

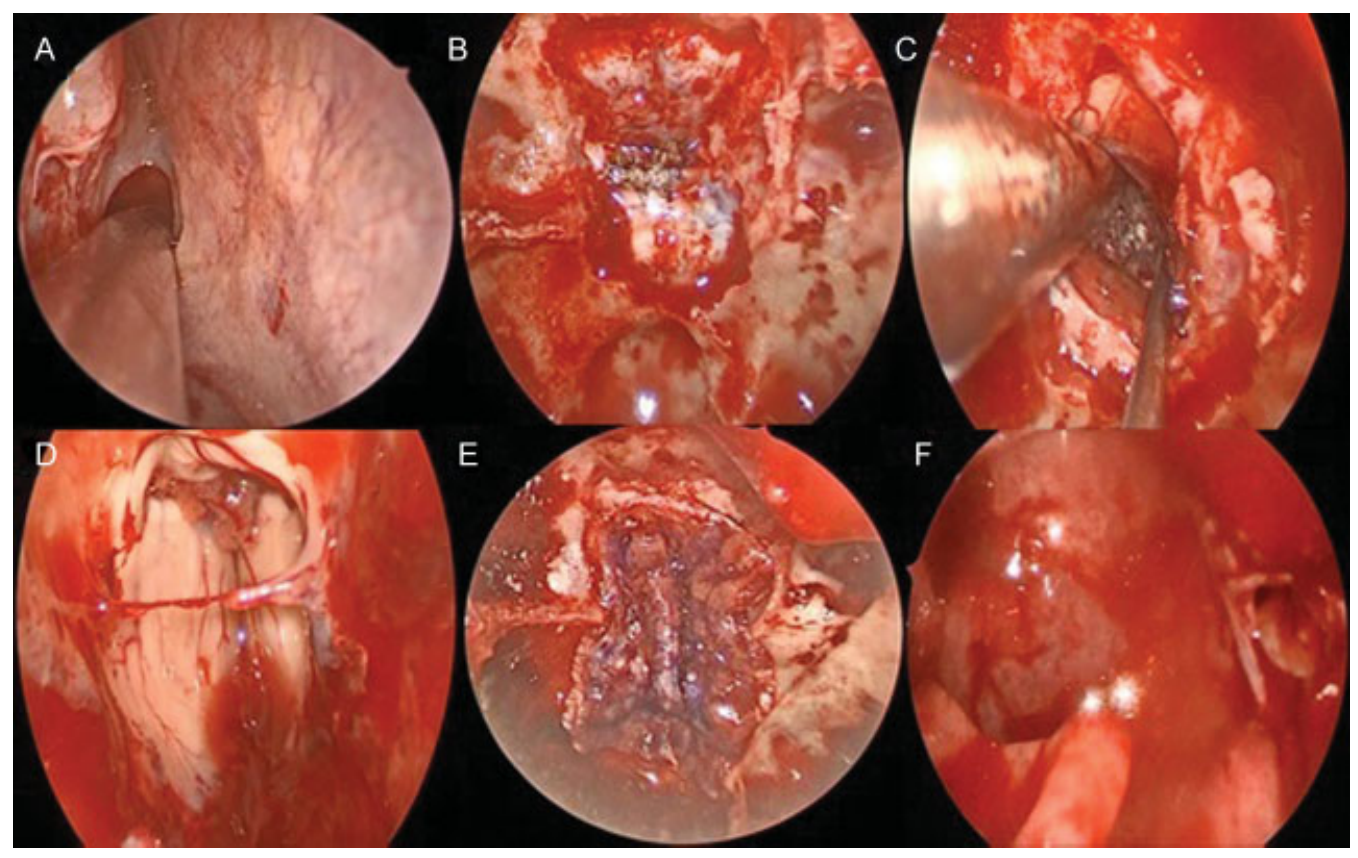

Fig. 2 Intraoperative images showing the identification and expansion of the sphenoid os (A), the bony opening over the sella and tuberculum (B), calcifications in the suprasellar lesion (C), the resection cavity (D), the fascia lata graft sealing the dural opening (E), and the nasoseptal flap rotated into position $(\mathbf{F})$. 